Article

\title{
Antimicrobial Activity of Resveratrol Analogues
}

Malik Chalal ${ }^{1, \dagger}$, Agnès Klinguer ${ }^{2}$, Abdelwahad Echairi ${ }^{3}$, Philippe Meunier ${ }^{4}$, Dominique Vervandier-Fasseur ${ }^{4}$ and Marielle Adrian ${ }^{1, *}$

1 Université de Bourgogne, UMR1347 Agroécologie, ERL CNRS 6300, BP 86510, 21065 Dijon Cedex, France; E-Mail: malik.chalal@univ-reims.fr

2 INRA, UMR1347 Agroécologie, ERL CNRS 6300, BP 86510, 21065 Dijon Cedex, France;

E-Mail: agnes.klinguer@dijon.inra.fr

3 Welience, Maison Régionale de L'Innovation, 64 A rue de Sully, CS 77124,

21071 Dijon Cedex, France; E-Mail: abdelwahad.echairi@welience.com

4 Institut de Chimie Moléculaire de l'Université de Bourgogne, ICMUB-UMR CNRS 6302,

9 Avenue Alain Savary, 21000 Dijon, France; E-Mails: Philippe.Meunier@u-bourgogne.fr (P.M.);

Dominique.Vervandier-Fasseur@u-bourgogne.fr (D.V.-F.)

$\dagger$ Present address: Institut de Chimie Moléculaire de Reims, UMR CNRS 7312, Campus du Moulin de la House, BP 1039, F-51687 Reims Cedex, France.

* Author to whom correspondence should be addressed; E-Mail: marielle.adrian@u-bourgogne.fr; Tel.: +33-(0)-3-80-69-34-85; Fax: +33-(0)-3-80-69-32-24.

Received: 25 April 2014; in revised form: 3 June 2014 / Accepted: 5 June 2014 /

Published: 10 June 2014

Abstract: Stilbenes, especially resveratrol and its derivatives, have become famous for their positive effects on a wide range of medical disorders, as indicated by a huge number of published studies. A less investigated area of research is their antimicrobial properties. A series of 13 trans-resveratrol analogues was synthesized via Wittig or Heck reactions, and their antimicrobial activity assessed on two different grapevine pathogens responsible for severe diseases in the vineyard. The entire series, together with resveratrol, was first evaluated on the zoospore mobility and sporulation level of Plasmopara viticola (the oomycete responsible for downy mildew). Stilbenes displayed a spectrum of activity ranging from low to high. Six of them, including the most active ones, were subsequently tested on the development of Botrytis cinerea (fungus responsible for grey mold). The results obtained allowed us to identify the most active stilbenes against both grapevine pathogens, to compare the antimicrobial activity of the evaluated series of stilbenes, and to 
discuss the relationship between their chemical structure (number and position of methoxy and hydroxy groups) and antimicrobial activity.

Keywords: resveratrol; stilbenes; grapevine; downy mildew; grey mold; Plasmopara viticola; Botrytis cinerea

\section{Introduction}

Plants possess an innate immune system that prevents their infection by most of microorganisms such as oomycetes and fungi [1]. This self-defense potential includes the production of the secondary metabolites phytoalexins, antimicrobial compounds synthesized and accumulated in response to biotic or abiotic stresses [2,3]. In grapevines, they are stilbenes synthesized via the phenylalanine/polymalonate pathway [4]. The key compound resveratrol (3,5,4'-trihydroxystilbene) is formed by condensation of one molecule of $p$-coumaroyl-CoA and three molecules of malonyl-CoA by stilbene synthase (EC 2.3.1.95). Subsequent glycosylation, methoxylation or dimerization reactions provides a spectrum of resveratrol derivatives [5]. Such modifications are essential for the biological activity of the so- formed compounds [6-8].

A huge number of works has reported the role of stilbenes, especially resveratrol, in human health. They have attracted attention for their high preventive or curative effects on a wide range of medical disorders and are known as cardioprotective, antitumor, neuroprotective and antioxidant agents (for reviews, see [9-13]). In comparison, the antimicrobial properties of stilbenes have been less investigated. In grapevines, stilbenes are constitutively accumulated at high concentrations in the heartwood where they act as phytoanticipins and can prevent the development of wood decay $[14,15]$. In other tissues, they are accumulated in response to various microorganisms including pathogens: Plasmopara viticola, Erysiphe necator, Botrytis cinerea, Phaeomoniella chlamydospora, Fusarium solani, Cladosporium cucumerinum, Pyricularia oryzae, Aspergilli, Rhizopus stolonifer (for review, see $[5,16,17])$. Whereas resveratrol generally shows a moderate antimicrobial activity, it is the precursor of more active derivatives such as pterostilbene and viniferins (for reviews, see [5,16,17]).

Recently, interest in the bioproduction and chemical synthesis of stilbenes has emerged to identify highly active molecules that could be used for medical applications and/or plant disease control [18-22]. Previous studies have shown that resveratrol is not the most active stilbene regarding antimicrobial activity [5,6,16,17]. In this study, 13 trans-resveratrol analogues were synthesized as previously described [20] to identify better candidates than trans-resveratrol to control two harmful grapevine pathogens: $P$. viticola (downy mildew) and B. cinerea (grey mold). This allowed us to compare their antimicrobial activity against both pathogens and to discuss the chemical structure/antimicrobial activity relationships of these compounds. 


\section{Results and Discussion}

\subsection{Activity of Stilbenes on P. viticola}

A series of 13 trans-resveratrol analogues was synthesized via Wittig or Heck reactions and the structures were confirmed by ${ }^{1} \mathrm{H},{ }^{13} \mathrm{C}-\mathrm{NMR}$, HRMS, and IR after purification [20]. The compounds differed by the number and position of hydroxy and/or methoxy groups (Figure 1).

Figure 1. Structure of the stilbenes used for bioassays. (a) 4-OH stilbenes bearing substituents on cycle B; (b) 4-OH stilbenes bearing substituents on cycle A and/or cycle B;

(c) Structure of 2-OH and 3-OH stilbenes; (d) Structure of stilbenes without phenolic function.

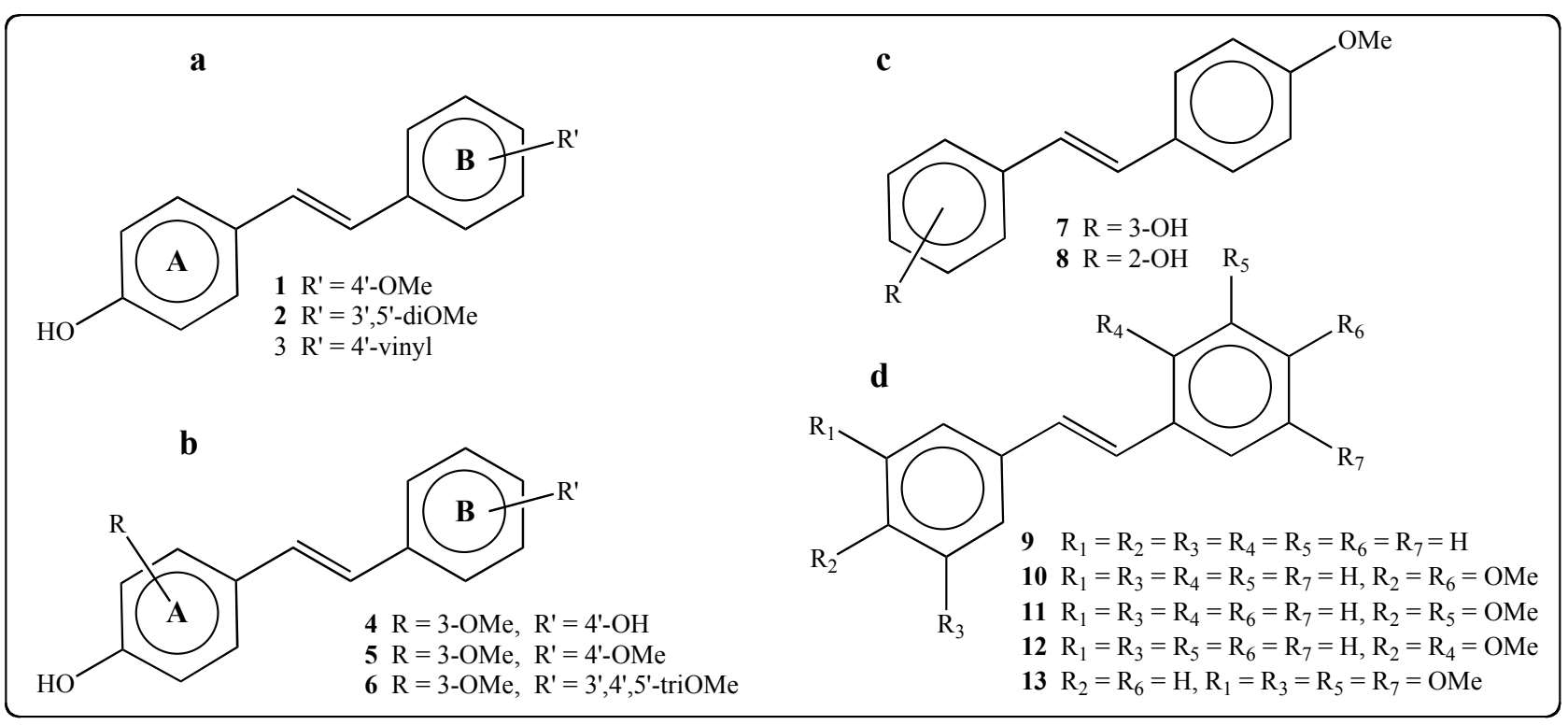

Their activity was first assessed in parallel with resveratrol against $P$. viticola, the oomycete responsible for grapevine downy mildew. This pathogen is obligatory and biotrophic i.e., it develops in leaving grapevine tissues and cannot be grown on artificial media. The assays were therefore performed by adding the compounds to a sporangia suspension $(0.25,0.5$ and $0.75 \mathrm{mM})$ prior to inoculation of leaf disks. The level of sporulation was determined by visual scoring at 6 days post inoculation as previously described [23]. Stilbenes were prepared in DMSO to ensure their dissolution and the final DMSO concentration in suspensions was $2 \%(\mathrm{v} / \mathrm{v})$. As there was interest in identifying stilbenes more active than resveratrol, the three concentrations assessed for this bioassay were chosen on the basis of resveratrol $\mathrm{ID}_{100}$ values towards $P$. viticola and/or $B$. cinerea previously reported in the literature [24-26]. The results obtained are presented in Figure 2.

DMSO alone only slightly reduced the level of sporulation in comparison with water control. All resveratrol analogues showed activity compared to both water and DMSO controls. The most active ones were 2, 4, 8, and resveratrol (RSV); especially $\mathbf{2}$ and RSV that totally inhibited the sporulation at all concentrations assessed. Compounds $\mathbf{6}$ and $\mathbf{1 2}$ were also highly active at 0.5 and $0.75 \mathrm{mM}$ and, to a lesser extent, compounds $\mathbf{3}$ and $\mathbf{7}$ at $0.75 \mathrm{mM}$. Compound $\mathbf{2}$ corresponds to pterostilbene, previously reported as having the highest antimicrobial activity among natural resveratrol derivatives $[6,25,26]$. In most cases, the presence of lateral groups increases the activity against $P$. viticola. For disubstituted 
stilbenes, the nature of the groups (-OH and -OMe vs. -OMe and -OMe) is important for activity although their position looks essential (comparisons 1 vs. 10, 7 vs. 11, 8 vs. 12). The presence of a methoxy group in position 4' together with a methoxy or hydroxy group in position 2 confer high activity. Resveratrol and tri-substituted derivatives were the most active compounds, suggesting the importance of the hydroxy group at position 4'. The presence of more than three groups does not increase activity.

Figure 2. Effect of stilbenes $(0.25,0.5$ and $0.75 \mathrm{mM})$ on $P$. viticola sporulation. Leaf disks (10/condition) were inoculated with a $P$. viticola sporangia suspension added by the stilbenes, water or DMSO $(2 \% \mathrm{v} / \mathrm{v}$ final concentration) as controls. The index of sporulation was scored at 6 days post-inoculation on a scale of 0 to 4 , where $0=$ no visible sporulation, $1=1 \%$ to $25 \%, 2=26 \%$ to $50 \%, 3=51 \%$ to $75 \%$, and $4=76 \%$ to $100 \%$ of the disk area covered. Values represent the mean index from three independent experiments.

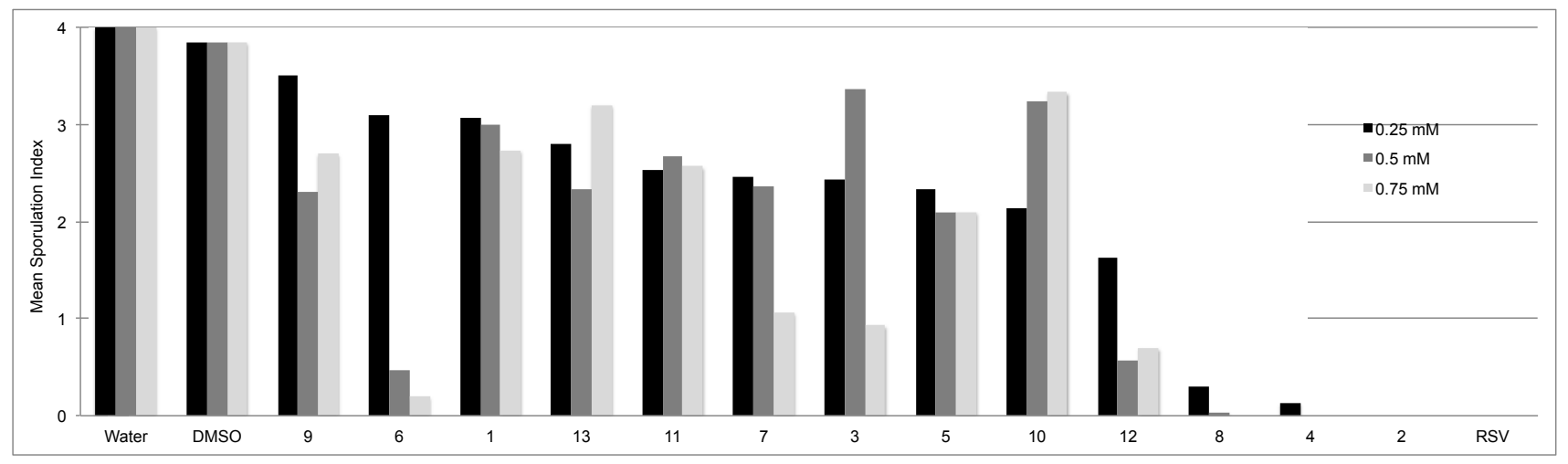

A prerequisite for successful downy mildew infection is zoospore mobility. Hence, once released by sporangia, bi-flagellated zoospores have to swim into water droplets present at the leaf surface to reach stomata where they encyst and form a germ tube that uses these natural pores to enter the leaf. The effects of the most active stilbenes were therefore measured on zoospore mobility (Table 1).

Table 1. Effects of stilbenes (RSV and compounds 2, 4, 6 and 8) on the mobility of $P$. viticola zoospores. Values correspond to the percentage of mobile zoospores released by sporangia in a suspension added by stilbenes $(0.25,0.5$, and $0.75 \mathrm{mM})$. The value of 100 was attributed to the number of mobile zoospores determined in the water control.

\begin{tabular}{cccc}
\hline Compound & $\mathbf{0 . 2 5} \mathbf{~ m M}$ & $\mathbf{0 . 5} \mathbf{~ m M}$ & $\mathbf{0 . 7 5} \mathbf{~ M}$ \\
\hline 2 & 0 & 0 & 0 \\
8 & 0 & 0 & 0 \\
$\mathrm{RSV}$ & 38 & 0 & 0 \\
4 & 38 & 23 & 0 \\
6 & 46 & 15 & 0 \\
\hline
\end{tabular}

For these bioassays, DMSO slightly reduced the mobility of zoospores $(90 \%$ of mobile zoospores towards the water control) and the most active compounds were $\mathbf{2}$ and $\mathbf{8}$. Curiously, no mobile zoospores could be observed in the presence of compound $\mathbf{8}$ whereas sporulation occured at $0.25 \mathrm{mM}$ and, to a lesser extent, at $0.5 \mathrm{mM}$. The mobility of zoospores in these conditions is maybe too low to 
be measured but sufficient to allow few zoospores to reach stomata and initiate infection. The opposite was observed for RSV at $0.25 \mathrm{mM}$ for which mobile zoospores were observed whereas no sporulation could be detected. Mobile zoospores might reach stomata but they could not germinate. The effects of natural stilbenes on the mobility of zoospores have been previously reported [26]. Resveratrol was less active than pterostilbene, as in our conditions, and also than viniferins.

\subsection{Activity of Stilbenes on B. cinerea}

We were interested in identifying stilbenes with high antimicrobial activity against both grapevine pathogens. On the basis of the results obtained with $P$. viticola, compounds 2, 4, 6, and 8 were evaluated against $B$. cinerea, the necrotroph fungus responsible for grey mold. Compounds $\mathbf{1}$ and $\mathbf{7}$ were included to allow comparison with $\mathbf{8}$. Conidia suspensions were prepared in the culture medium and added by stilbenes or DMSO ( $2 \% \mathrm{v} / \mathrm{v}$ final concentration) as control. Bioassays were performed in microplates and the mycelium development was automatically recorded at regular time intervals by spectrophotometry for $60 \mathrm{~h}$. Stilbenes were tested at concentrations ranging from 0.01 to $0.75 \mathrm{mM}$ to allow the determination of their $\mathrm{IC}_{50}$ value (Table 2). As previous studies have clearly shown that resveratrol is less active than pterostilbene (2) against $B$. cinerea $[5,6,16,17,25]$ it was not included in these assays at different concentrations but only at $0.5 \mathrm{mM}$ as positive control. The $\mathrm{IC}_{50}$ of pterostilbene was therefore used for comparison. Resveratrol $(0.5 \mathrm{mM})$ was systematically included in each bioassay as reference.

Table 2. Effects of stilbenes on $B$. cinerea development. Values correspond to the concentration that inhibits $50 \%$ of the mycelial growth $\left(\mathrm{IC}_{50}\right)$. SE: Standard error.

\begin{tabular}{cc}
\hline Compound & $\mathbf{I C}_{\mathbf{5 0}} \pm \mathbf{S E}(\boldsymbol{\mu M})$ \\
\hline 7 & $28 \pm 3$ \\
8 & $30 \pm 5$ \\
2 & $52 \pm 4$ \\
4 & $55 \pm 11$ \\
1 & $>100$ \\
6 & $>100$ \\
\hline
\end{tabular}

DMSO alone did not inhibit the mycelial development (data not shown). Compound 8 appeared highly interesting since it showed high antimicrobial activity against both pathogens. It was more efficient than pterostilbene (2), generally described as the most active resveratrol analogue, on $B$. cinerea. This highlights the interest of this disubstituted stilbene. According to the results obtained with $P$. viticola, compound $\mathbf{4}$ also showed a high activity whereas compounds $\mathbf{1}$ and $\mathbf{6}$ were less active. Interestingly, compound 7 showed a high antimicrobial activity on $B$. cinerea but was less effective on $P$. viticola. Altogether, these results confirm that the chemical structure of stilbenes is essential for their biological activity. As example, resveratrol methylation confers a higher antifungal activity $[6,19,25]$. Monoglucosides also exhibit an antioxidant activity that depends on the location of the hydroxy groups and the type of the sugar residue [7]. Albert et al. [19] also reported this structure/biological activity relationship. However, in some cases, it depends on microorganisms. 
As example, they observed that the activity of methoxylated stilbenes was significant for fungi but low for bacteria. It would be interesting to determine what make the specificity of the activity of such compounds.

At the end of the experiments, the fungus was collected from the microplates and observed by microscopy. Characteristic images are presented in Figure 3. In control conditions, B. cinerea developed a thick network of long hyphae (Figure 3a). Conversely, in presence of active compounds $(\mathbf{2}, \mathbf{4}, \mathbf{7}$ and 8), the mycelium development was restricted (Figure 3b). One could observe short gem tubes and thinned hyphal tips, indicating that the fungal development was stopped. In some cases, dead conidia released their intercellular content (Figure 3c, arrow). These observations are typical of the toxic effects of stilbenes [25,27].

Figure 3. Representative photographs showing B. cinerea mycelial development (a) in the culture medium alone as control or (b,c) added by compounds 2, 4, 7 or $\mathbf{8}$. Observations were made using a Leitz DMRB microscope.
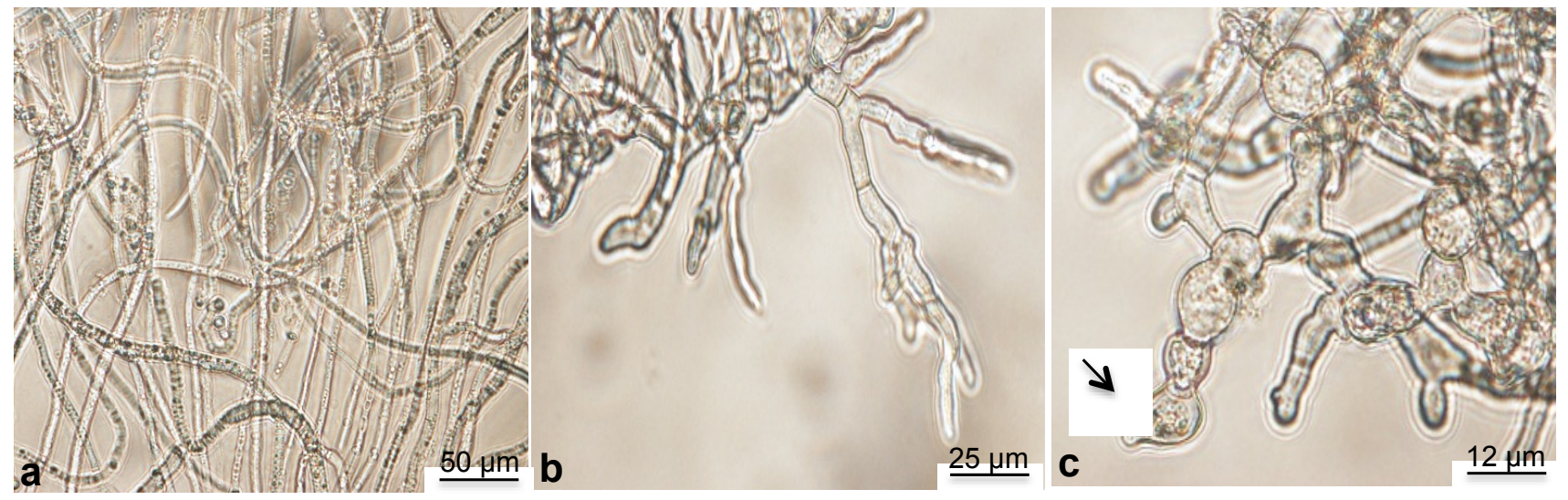

\section{Experimental}

\subsection{Synthesis}

The syntheses and characterization of the 13 stilbenes assessed were previously reported [20]. Briefly, preparation of compounds 4-8, and 10-12 was achieved using palladium catalysis Heck coupling reactions. Stilbenes $\mathbf{1}-\mathbf{3}, \mathbf{1 0}$ and $\mathbf{1 3}$ were prepared through classic Wittig reactions.

\subsection{Plant Material}

Grapevine ( $V$. vinifera L. cv. Marselan) herbaceous cuttings were grown in a glasshouse at a temperature of 24 and $18{ }^{\circ} \mathrm{C}$ (day and night, respectively), with a photoperiod of $16 \mathrm{~h} \mathrm{light,} \mathrm{and} \mathrm{at} \mathrm{a}$ relative humidity $(\mathrm{RH})$ of $70 \% \pm 10 \%$, as previously described [28]. Once they developed six leaves, the second and third leaves below the apex were excised and used for $P$. viticola inoculation. 


\subsection{P. viticola Bioassays}

\subsubsection{Assessment of Stilbene Effects on Sporulation}

The $P$. viticola strain was maintained on Marselan plants as previously described [28]. For bioassays, leaf disks (1 cm diameter) were punched out and placed in a Petri dish on wet filter paper. They were inoculated with $30 \mu \mathrm{L}$ of a $10^{5}$ sporangia/mL suspension prepared in distilled water and added by stilbenes dissolved in DMSO $(0.25,0.5$ and $0.75 \mathrm{mM}, 2 \% \mathrm{v} / \mathrm{v}$ final concentration of DMSO). The suspension was also added by water and DMSO alone ( $2 \% \mathrm{v} / \mathrm{v}$ final concentration) as controls. The antimicrobial activity of compounds was determined 6 days post inoculation (dpi) by visual scoring of the index of sporulation on a scale of 0 to 4 , where $0=$ no visible sporulation, $1=1 \%$ to $25 \%, 2=26 \%$ to $50 \%, 3=51 \%$ to $75 \%$, and $4=76 \%$ to $100 \%$ of the disk area covered. Ten leaf disks were prepared per condition and the experiment was repeated three times.

\subsubsection{Assessment of Stilbene Effects on Zoospore Mobility}

Assays were performed using a modified method previously described [26]. A sporangia suspension of $P$. viticola was prepared at $5 \times 10^{4}$ sporangia $\cdot \mathrm{mL}^{-1}$ in distilled water and added by stilbene dissolved in DMSO $(0.25,0.5$ and $0.75 \mathrm{mM}, 2 \% \mathrm{v} / \mathrm{v}$ final concentration of DMSO). The suspension was also added by water and DMSO (2\% v/v final concentration) alone as controls. After $45 \mathrm{~min}$ at room temperature with frequent and gentle handly stirring to allow the release of zoospores, $30 \mu \mathrm{L}$ of the suspension was deposited on a Malassez cell. The number of zoospores passing through an area of one rectangle of the cell was determined during 1 min using a microscope (magnification $\times 40$ ).

\subsection{B. cinerea Bioassays}

A conidial suspension of the $B$. cinerea strain BMM was prepared at $2 \times 10^{5}$ conidia/mL of PDB (Potato Dextrose Broth 1/4) medium. Stilbenes prepared in DMSO were added to the suspension (at $0.01,0.02,0.05,0.1,0.25$ and $0.5 \mathrm{mM}, 2 \% \mathrm{v} / \mathrm{v}$ final concentration of DMSO). The suspension was also added by DMSO alone ( $2 \% \mathrm{v} / \mathrm{v}$ final concentration) as control. One hundred microliters of each suspension were distributed in microplates for automatic spectrophotometry recording (Bioscreen C, Thermoelectron Led, St Herblain, France). The absorbance at $492 \mathrm{~nm}$ was measured and recorded every $2 \mathrm{~h}$ for $60 \mathrm{~h}$.

\section{Conclusions}

This study allowed us to compare the antimicrobial activity of $13 E$-stilbenes with resveratrol on two grapevine pathogens. Altogether, the results confirm the importance of the chemical structure of stilbenes regarding their biological activity. However, they did not allow us to draw a clear and direct relationship between the structure of a compound (number/position of $\mathrm{OH}$ - and $\mathrm{OCH}_{3}$ - groups) and its antimicrobial activity, making it difficult to predict the level of antimicrobial activity of a stilbene. The results also highlight that the activity of resveratrol analogues is microorganism dependent. Among the 13 trans-resveratrol derivatives assessed, only three of them were highly active against both pathogens. One of them is the already known pterostilbene. The other ones are 2-hydroxy, 
4-methoxystilbene (8) and 3-methoxy-4,4'-hydroxystilbene (4). Compound 7 (3-hydroxy-4'methoxystilbene) also looks interesting, but only against $B$. cinerea. Interestingly, compounds 7 and 8 showed a higher antimicrobial activity against $B$. cinerea, compared to the highly active pterostilbene. It would be interesting to investigate the biological activity of these new compounds against a larger spectrum of microorganisms.

\section{Acknowledgments}

We thank the Conseil Régional de Bourgogne and Bureau Interprofessionnel des Vins de Bourgogne for financial support, Adrian Variot for technical assistance, and Arnaud Haudrechy for helpful comments concerning the manuscript. Malik Chalal benefited of a $\mathrm{PhD}$ grant from Algerian government (Ministère de la Recherche et de l'Enseignement), which is sincerely acknowledged.

\section{Author Contributions}

MA, DVF and PM designed research; $\mathrm{MC}, \mathrm{AK}$ and $\mathrm{AE}$ performed research, $\mathrm{MA}, \mathrm{MC}$ and $\mathrm{AE}$ analyzed the data; MA, MC and DVF wrote the paper. All authors read and approved the final manuscript.

\section{Conflicts of Interest}

The authors declare no conflict of interest.

\section{References}

1. Nürnberger, T.; Brunner, F.; Kemmerling, B.; Piater, L. Innate immunity in plants and animals: Striking similarities and obvious differences. Immunol. Rev. 2004, 198, 249-266.

2. Kuc, J. Phytoalexins, stress metabolism, and disease resistance in plants. Annu. Rev. Phytopathol. 1995, 33, 275-297.

3. Jeandet, P.; Clément, C.; Courot, E.; Cordelier, S. Modulation of phytoalexin biosynthesis in engineered plants for disease resistance. Int. J. Mol. Sci. 2013, 14, 14136-14170.

4. Langcake, P.; Pryce, R.J. The production of resveratrol by Vitis vinifera and other members of the Vitaceae as a response to infection or injury. Physiol. Mol. Plant Pathol. 1976, 9, 77-86.

5. Chong, J.L.; Poutaraud, A.; Hugueney, P. Metabolism and roles of stilbenes in plants. Plant Sci. 2009, 177, 143-155.

6. Pont, V.; Pezet, R. Relation between the chemical structure and the biological activity of hydroxystilbenes against Botrytis cinerea. J. Phytopathol. 1990, 130, 1-8.

7. Orsini, F.; Pellizzoni, F.; Verotta, L.; Aburjai, T.; Rogers, C.B. Isolation, synthesis, and antiplatelet aggregation activity of resveratrol 3-o-beta-d-glucopyranoside and related compounds. J. Nat. Prod. 1997, 60, 1082-1087.

8. Regev-Shoshani, G.; Shoseyov, O.; Bilkis, I.; Kerem, Z. Glycosylation of resveratrol protects it from enzymic oxidation. Biochem. J. 2003, 374, 157-163. 
9. Aggarwal, B.B.; Bhardwaj, A.; Aggarwal, R.S.; Seeram, N.P.; Shishodia, S.; Takada, Y. Role of resveratrol in prevention and therapy of cancer: Preclinical and clinical studies. Anticancer Res. 2004, 24, 2783-2840.

10. Baur, J.A.; Sinclair, D.A. Therapeutic potential of resveratrol: The in vivo evidence. Nat. Rev. Drug Discov. 2006, 5, 493-506.

11. Roupe, K.A.; Remsberg, C.M.; Yanez, J.A.; Davies, N.M. Pharmacometrics of stilbenes: Seguing towards the clinic. Curr. Clin. Pharmacol. 2006, 1, 81-101.

12. Patel, K.R.; Scott, E.; Brown, V.A.; Gescher, A.J.; Steward, W.P.; Brown, K.; Ann, N.Y. Clinical trials of resveratrol. Acad. Sci. 2011, 1215, 161-169.

13. Pezzuto, J.M. The phenomenon of resveratrol: Redefining the virtues of promiscuity. Ann. N. $Y$. Acad. Sci. 2011, 1215, 123-130.

14. Hart, J.H.; Shrimpton, D.M. Role of stilbenes in resistance of wood to decay. Phytopathology 1979, 69, 1138-1143.

15. Hart, H. Role of phytostilbenes in decay and disease resistance. Annu. Rev. Phytopathol. 1981, 19, 437-458.

16. Jeandet, P.; Delaunois, B.; Conreux, A.; Donnez, D.; Nuzzo, V.; Cordelier, S.; Clément, C.; Courot, E. Biosynthesis, metabolism, molecular engineering, and biological functions of stilbene phytoalexins in plants. Biofactors 2010, 36, 331-341.

17. Adrian, M.; de Rosso, M.; Bavaresco, L.; Poinssot, B.; Héloir, M.C. Resveratrol from vine to wine. In Resveratrol Sources, Production and Health benefits; Delmas, D., Ed.; Nova Science Publishers Inc.: New York, NY, USA, 2013; pp. 3-19.

18. Mazue, F.; Colin, D.; Gobbo, J.; Wegner, M.; Rescifina, A.; Spatafora, C.; Fasseur, D.; Delmas, D.; Meunier, P.; Tringali, C.; et al. Structural determinants of resveratrol for cell proliferation inhibition potency: Experimental and docking studies of new analogs. Eur. J. Med. Chem. 2010, 45, 2972-2980.

19. Albert, S.; Horbach, R.; Deising, H.B.; Siewert, B.; Csuk, R. Synthesis and antimicrobial activity of (E) stilbene derivatives. Bioorg. Med. Chem. 2011, 19, 5155-5166.

20. Chalal, M.; Vervandier-Fasseur, D.; Meunier, P.; Cattey, H.; Hierso, J.C. Syntheses of polyfunctionalized resveratrol derivatives using Wittig and Heck protocols. Tetrahedron Lett. 2012, 68, 3899-3907.

21. Bhusainahalli, V.M.; Spatafora, C.; Chalal, M.; Vervandier-Fasseur, D.; Meunier, P.; Latruffe, N.; Tringali, C. Resveratrol-related dehydrofimers: Laccase mediated biomimetic synthesis and antiproliferative activity. Eur. J. Org. Chem. 2012, 27, 5217-5224.

22. Jeandet, P.; Vasserot, Y.; Chastang, T.; Courot, E. Engineering microbial cells for the biosynthesis of natural compounds of pharmaceutical significance. BioMed Res. Int. 2013, 2013, 780145:1-780145:13.

23. Trouvelot, S.; Varnier, A.L.; Allègre, M.; Mercier, L.; Baillieul, F.; Arnould, C.; Gianinazzi-Pearson, V.; Klarzynski, O.; Joubert, J.M.; Pugin, A.; et al. A beta-1,3 glucan sulfate induces resistance in grapevine against Plasmopara viticola through priming of defense responses, including HR-like cell death. Mol. Plant Microbe Int. 2008, 21, 232-243.

24. Langcake, P. Disease resistance of Vitis spp. and the production of the stress metabolites resveratrol, $\varepsilon$-viniferin, $\alpha$-viniferin and pterostilbene. Physiol. Plant Pathol. 1981, 18, 213-226. 
25. Adrian, M.; Jeandet, P.; Veneau, J.; Weston, L.A.; Bessis, R. Biological activity of resveratrol, a stilbenic compound from grapevines, against Botrytis cinerea, the causal agent for gray mold. J. Chem. Ecol. 1997, 23, 1689-1702.

26. Pezet, R.; Gindro, K.; Viret, O.; Richter, H. Effect of resveratrol, viniferins and pterostilbene on Plasmopara viticola zoospore mobility and disease development. Vitis 2004, 43, 145-148.

27. Adrian, M.; Jeandet, P. Effects of resveratrol on the ultrastructure of Botrytis cinerea conidia and biological significance in plant/pathogen interactions. Fitoterapia 2012, 83, 1345-1350.

28. Allègre, M.; Héloir, M.C.; Trouvelot, S.; Daire, X.; Pugin, A.; Wendehenne, D.; Adrian, M. Are grapevine stomata involved in the elicitor-induced protection against downy mildew? Mol. Plant Microbe Int. 2009, 22, 977-986.

Sample Availability: Samples of the compounds are not available.

(C) 2014 by the authors; licensee MDPI, Basel, Switzerland. This article is an open access article distributed under the terms and conditions of the Creative Commons Attribution license (http://creativecommons.org/licenses/by/3.0/). 